\title{
A Kalman Filter based Sway Velocity Estimation for Rudder Roll Control of Ships
}

\author{
Radhakrishnan K \\ Mar Athanasius College of Engineering \\ Kothamangalam, Kerala, PIN- 686666
}

\begin{abstract}
The sway velocity, which represents the interaction between sway, roll and yaw of ship, is not actually measurable. However, for effective control of the ship, this interaction also is to be taken into account. In this paper, we present a kalman filter approach for the estimation of the sway velocity, which is one of the states of the mathematical model describing the dynamics of the ship in headsets. The effect of the high frequency rudder motion, used to stabilize the roll on the heading of the ship is determined by simulation results.
\end{abstract}

\section{General Terms}

Parameter estimation, System Identification

\section{Keywords}

Kalman filter, rudder roll control

\section{INTRODUCTION}

The motions of ships and the control of those motions have been the focal point of extensive research over the years. A ship in a seaway undergoes complex motions that may reduce the operational range of the ship. A ship freely floating is subjected to 6 degrees of freedom, of which heave(Z), Sway $(\mathrm{Y})$, Surge $(\mathrm{X})$ are transational and Yaw(Z),Pitch(Y), Roll(X) are rotational.as shown in figure 1. There are numerous reasons for trying to control and reduce the motions of a ship[1].

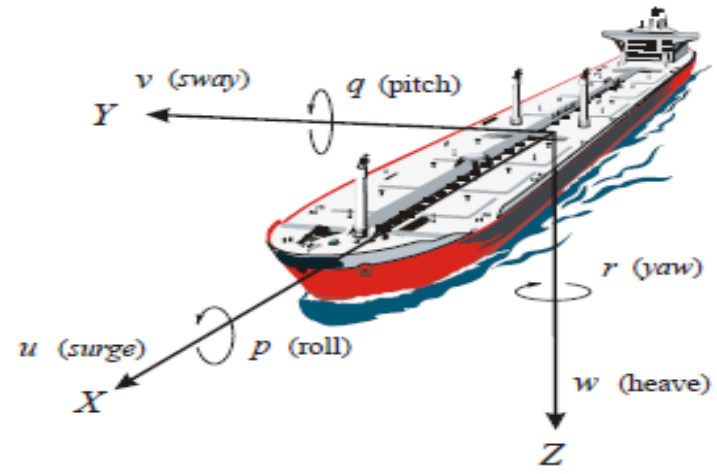

\section{Figure 1. Translational and rotational motions of a ship}

Excessive motions can interfere with the activities of the crew or passengers, reduce the combat readiness of naval vessels, cause the loss of containers on cargo vessels, and reduce the operational parameters of various ships. Over the years, many types of motion control or reduction devices have been devised. Most have been aimed at reducing roll motions since the force required to reduce roll is reasonably small compared to the weight of the ship. Moreover, roll is the largest and most undesirable component of ship motion. Various methods include gyroscopic stabilizers, anti-roll tanks, fins, moving weights, and active rudder control. Most of these methods first started as passive control and then evolved into active control.

Roll reduction in passenger and naval ships is an important area of research. Rudder Roll Reduction Control System ( RRCS ) have been designed for effective control of roll. But there is cross-coupling between sway, roll, and yaw[2]. For example, high frequency rudder motion is used for roll stabilization. Nevertheless, this high frequency rudder motion disturbs the heading control system. In order to reduce the interaction, models describing the coupling between the rudder angle to the linear and angular velocity in sway and yaw are used. The roll angle $\Phi$ and the heading angle $\Psi$ can be measured by gyros and their derivatives can be measured by rate gyros. However, to obtain full state feedback, the sway velocity $\mathrm{V}$ due to the rudder cannot be measured directly. This paper investigates the use of kalman filter for estimating the sway velocity.

\section{KALMANFILTER EQUATIONS}

Kalman filter is an optimal state estimator in the sense of minimum variance[3]-[4]. It allows estimating the state $X$ of a dynamic system with noise described by using a time sequence of measurements of the system behaviour plus a statistical model that characterizes the system and measurement error plus initial condition information. The important equations are

$$
X(k+1)=A X(k)+B U(k)+G W(k)
$$

Where $\mathrm{X}(\mathrm{k})=$ the state vector and $\mathrm{U}(\mathrm{k})=$ the control vector

$Y(k)=C X(k)+V(k)$

Where $\mathrm{Y}(\mathrm{k})$ - the output Vector, $\mathrm{W} \backsim(0, \mathrm{Q})$ - process noise which represents disturbances or modeling in accuracies which is considered as a stationary white noise process with zero mean and covariance $\mathrm{Q}$. $\mathrm{Vk} \sim(0, \mathrm{R})$ - measurement noise with zero mean and covariance $\mathrm{R}$. The kalman filter equations given by (1) and (2) can be derived as follows. 
$\hat{X}(k, k-1)=A(k-1) X(k-1, k-1)$

$\hat{X}(k, k)=\hat{X}(k, k-1)+G(k) L$

Where

$L=Y(k)-C(k) X(k, k-1)$

$G(k)=P(k, k-1) C(k)^{T} S$

$S=\left[C(k) P(k, k-1) C(k)^{T}+R_{v}\right]^{-1}$

$P(k, k-1)=A(k-1) P(k-1, k-1) A(k-1)^{T}+Q_{W}$

$P(k, k)=[I-G(k) C(k) P(k, k-1)$

where

$\mathrm{P}(\mathrm{k}, \mathrm{k}-1)$ - state prediction covariance

S - measurement prediction covariance

$\mathrm{P}(\mathrm{k}, \mathrm{k}) \quad$ - covariance update

$\mathrm{X}(\mathrm{k}, \mathrm{k})$ - state update

G(k) - Kalman gain

L - residue or innovation

\section{MATHEMATICAL MODEL FOR RRCS DESIGN}

The analysis of ship wave interaction require simple but effective model. In ship/wave control, deals with the kalman filter in the context of control system. The filter serves as the component of the system such as a controller or detector[5][6]. The model of Vander Klugt (1987) has shown to be practical for rudder roll and autopilot control system design [2] which is shown in figure 2.

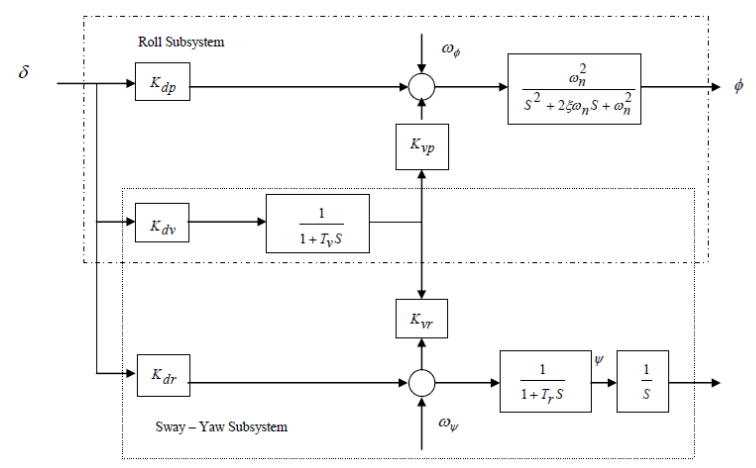

Figure 2. Cross coupling between sway-yaw and roll subsystems

The transfer functions are

$$
\dot{V}(s)=\frac{K_{d v} \delta(s)}{1+T_{v} S}
$$

$$
\phi(s)=\frac{\omega_{n}{ }^{2}\left[K_{d p} \delta(s)+K_{v p} \dot{V}(s)+\omega_{\phi}(s)\right]}{s^{2}+2 \xi \omega_{n}(s)+\omega_{n}{ }^{2}}
$$

$$
\psi(s)=\frac{\left[K_{d r} \delta(s)+K_{v r} \dot{V}(s)+\omega_{\psi}(s)\right]}{\left(1+T_{r} S\right) S}
$$

where $\dot{V}$ is a new state variable representing the sway velocity caused by the rudder motion alone. This state variable cannot be measured but a kalman filter can be used to estimate $V^{\prime}$. The signals $\omega_{\Phi}$ and $\omega_{\Psi}$ are included in the above model to describe wave-induced disturbances.

The corresponding state-space model is

$\dot{X}=A X+B u+G W$

Where

$X=[\dot{V}, r, \psi, P, \phi]$

$\mathrm{V}$ - Sway velocity, $\mathrm{r}$ - Yaw rate, $\Psi$ - heading angle (yaw angle), $\Phi$ - roll angle., $\mathrm{P}-$ Roll rate.

$\mathbf{A}=\left[\begin{array}{lllll}\frac{\mathbf{1}}{\mathbf{T} v} & \mathbf{0} & \mathbf{0} & \mathbf{0} & \mathbf{0} \\ \mathbf{K} v r & \frac{-\mathbf{1}}{\mathbf{T} r} & \mathbf{0} & \mathbf{0} & \mathbf{0} \\ \mathbf{0} & \mathbf{1} & \mathbf{0} & \mathbf{0} & \mathbf{0} \\ \boldsymbol{\omega}_{\mathbf{n}}{ }^{2} \mathbf{K} v p & \mathbf{0} & \mathbf{0} & -2 \xi \omega_{\mathbf{n}} & \boldsymbol{\omega}_{\mathbf{n}} \\ \mathbf{0} & \mathbf{0} & \mathbf{0} & \mathbf{1} & \mathbf{0}\end{array}\right]$

$$
\begin{aligned}
& B=\left[\begin{array}{lllll}
\frac{K d v}{T v} & \frac{K d r}{T r} & 0 & -\omega_{n}^{2} K d p & 0
\end{array}\right]^{T} \\
& W=\left[\begin{array}{ll}
\omega_{\phi} & \omega_{\psi}
\end{array}\right] T
\end{aligned}
$$

$u=\delta$ - the rudder angle

\section{SIMULATION RESULTS}

In this section we demonstrate the use of Kalman filtering for estimating the sway velocity and the effects of it's cross coupling between roll and yaw. The model parameters are $\omega_{\Phi}$ $=0.7, \omega_{\Psi}=0.35, \omega_{\mathrm{n}}=0.63, \mathrm{~T}_{\mathrm{v}}=78 / \mathrm{U}, \mathrm{T}_{\mathrm{r}}=13 / \mathrm{u}, \mathrm{K}_{\mathrm{vr}}=-$ $0.46, \mathrm{~K}_{\mathrm{dr}}=-0.0027 \mathrm{U}, \mathrm{K}_{\mathrm{dp}}=-0.0014 / \mathrm{U}^{2}, \mathrm{~K}_{\mathrm{dv}}=0.01 \mathrm{U}, \mathrm{K}_{\mathrm{vp}}=$ $0.21 \mathrm{U}, \xi=0.064+0.0038 \mathrm{U}$, where $\mathrm{U}$ is the forward speed .

Figure 1a shows the roll of the vessel in the absence of sway velocity feedback. For the first simulation example $8 \mathrm{~m} / \mathrm{sec}$ was used as ship speed and for the second example $15 \mathrm{~m} / \mathrm{sec}$ was used as the ship speed. The rudder input is assumed to be sinusoidal of amplitude 10 and frequency of $0.43 \mathrm{rad} / \mathrm{sec}$. Figs 
2(a)-2(g) show different states(ie roll, roll rate, yaw, yaw rate and sway velocity of the system and figs $2(\mathrm{~h})-2(\mathrm{~m})$ shows the results of second simulation. In these simulations using matlab[7] it was found that for the ship speed up to $13 \mathrm{~m} / \mathrm{sec}$ the roll is reduced to one- tenth of that without sway velocity

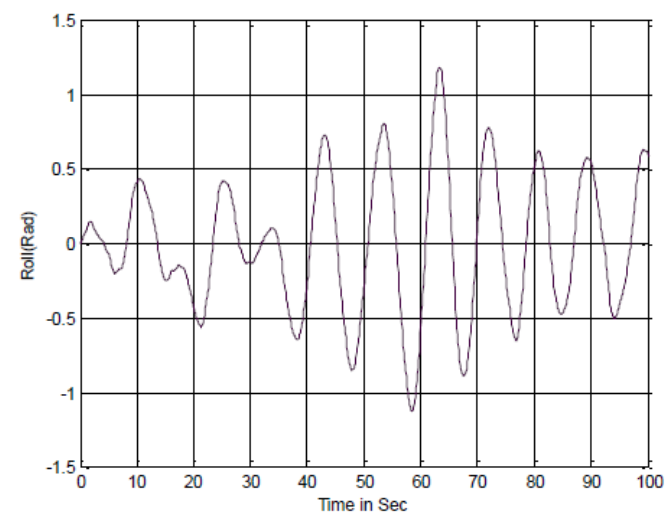

Figure 1a Variation of Roll in the absence of sway velocity

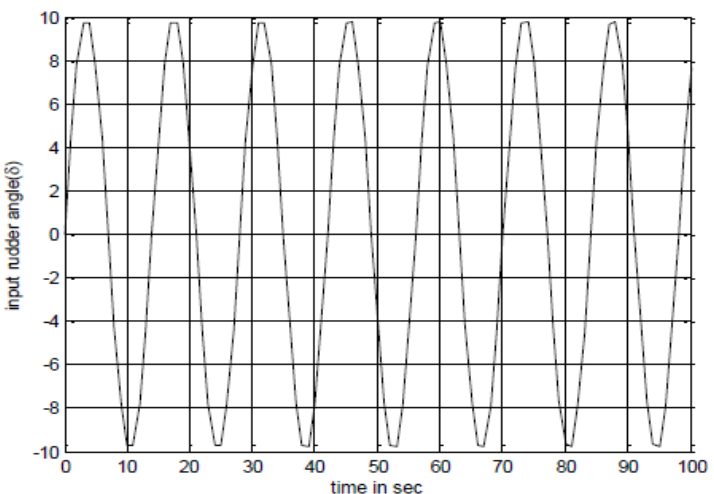

Figure 2a Sinusoidal rudder input of frequency 0.43 $\mathrm{rad} / \mathrm{sec}$

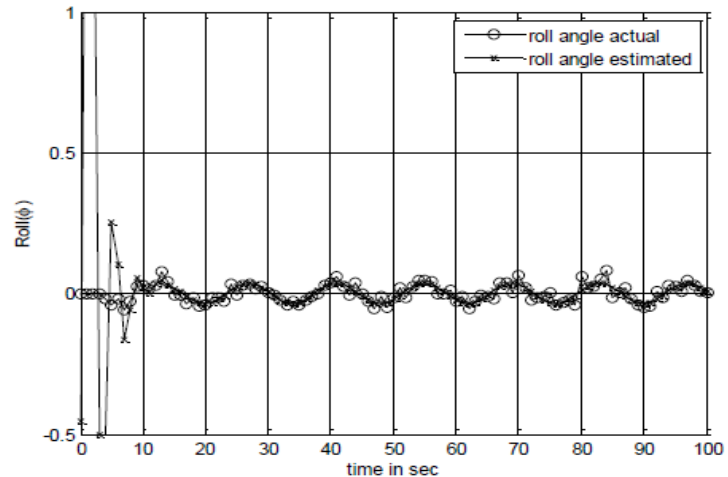

Figure $2 b$. Variation of actual and estimated roll angle feedback and the roll and yaw settle down to a reasonable tolerance level after few iterations. For $\mathrm{U}=15 \mathrm{~m} / \mathrm{sec}$ onwards it becomes oscillatory showing instability.

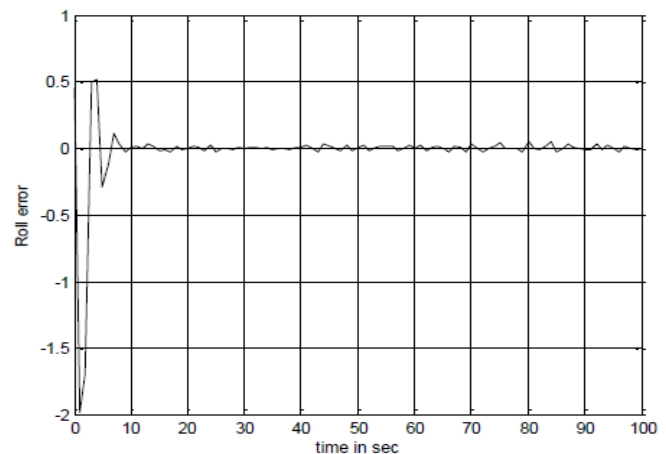

Figure2c Error in the estimated roll angle

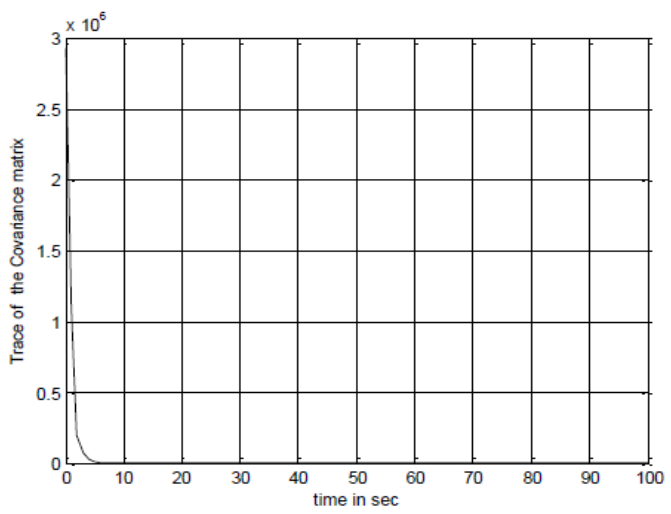

Figure2d Trace of the covariance matrix $P$ in estimationg Sway velocity

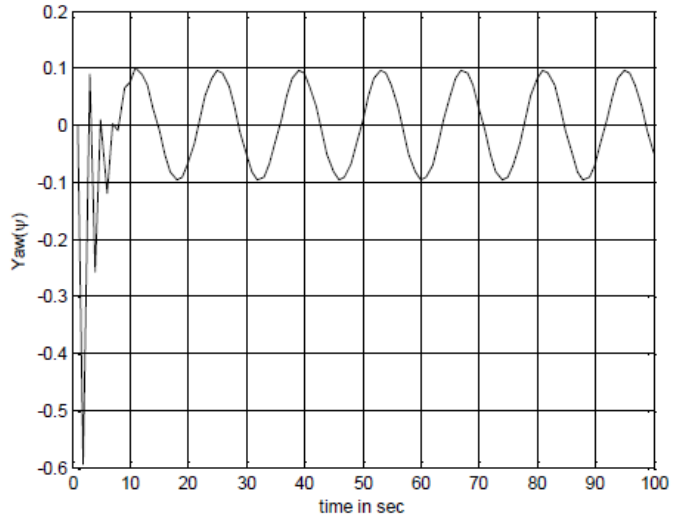

Figure 2e. Variation of Yaw angle 


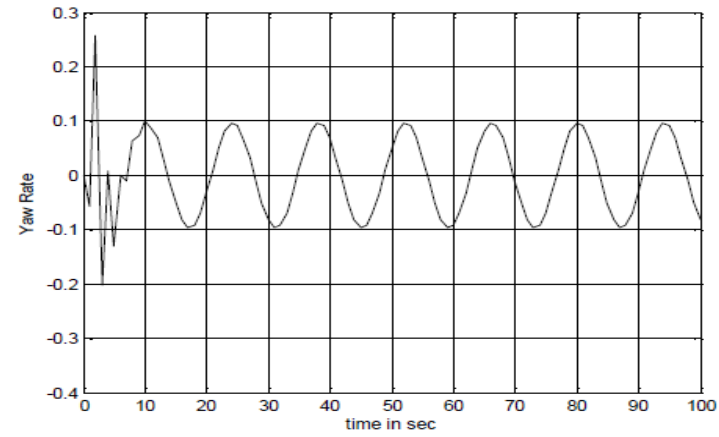

Figure 2f. Variation of Yaw rate

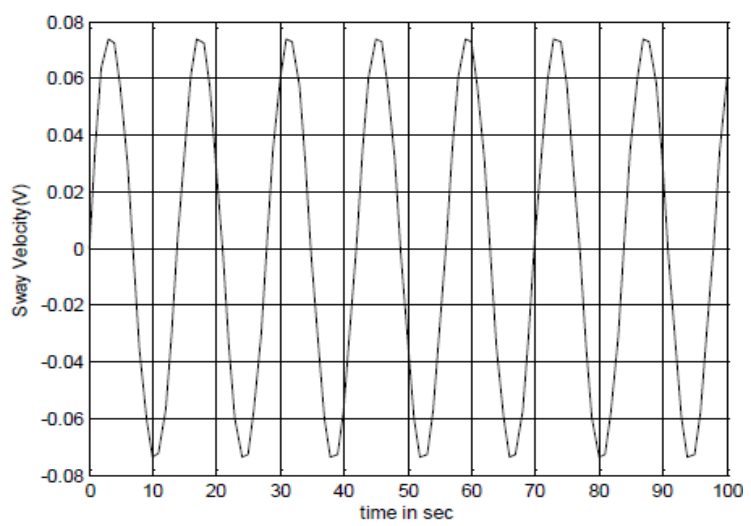

Figure2g Variation of sway velocity

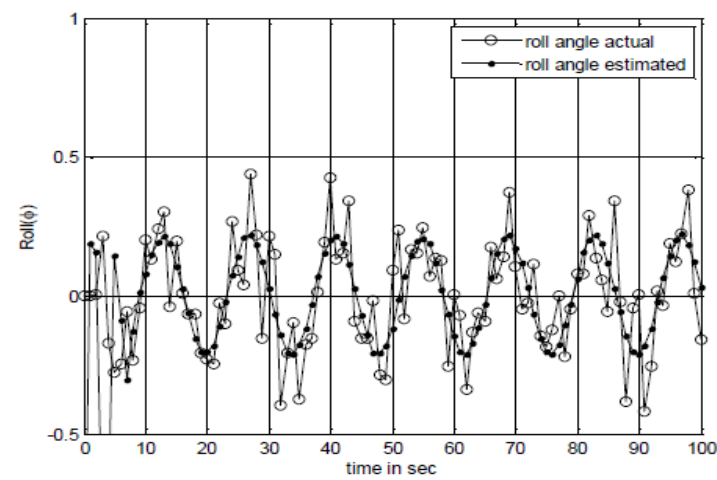

Figure $2 \mathrm{~h}$ Variation of actual and estimated roll angle

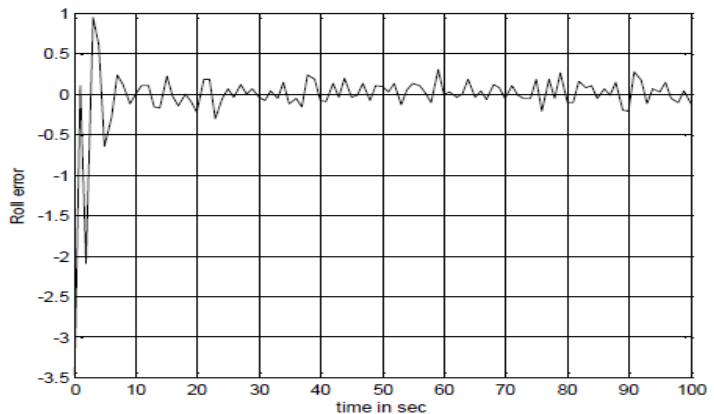

Figure 2i Error in estimated roll angle

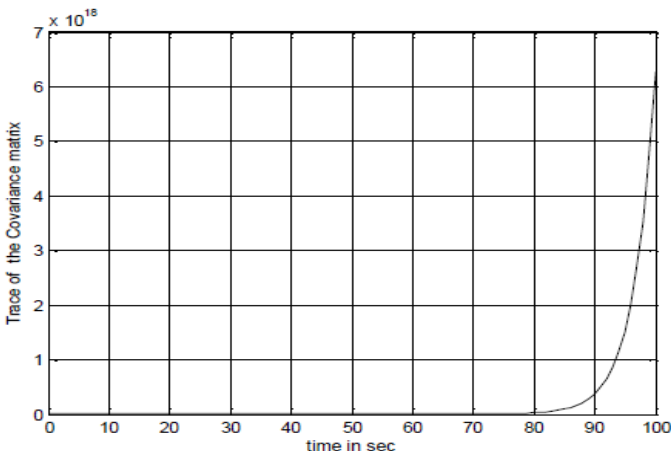

Figure 2j Trace of the covariance matrix $P$ in estimating Swav velocitv

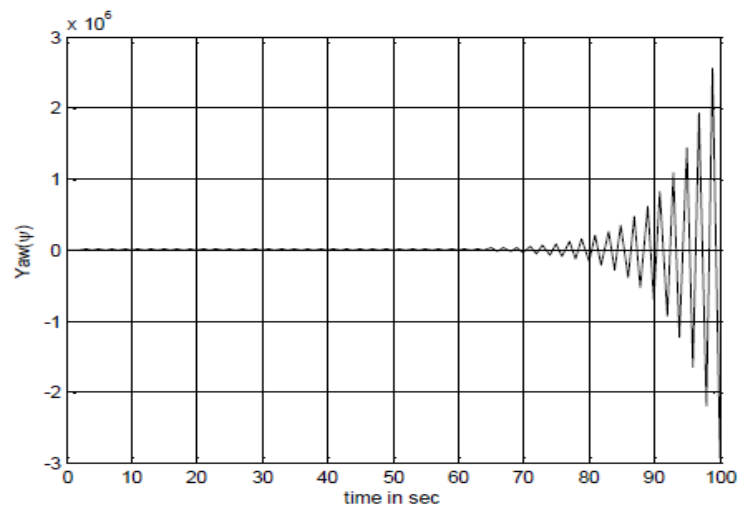

Figure 2k Variation of Yaw angle

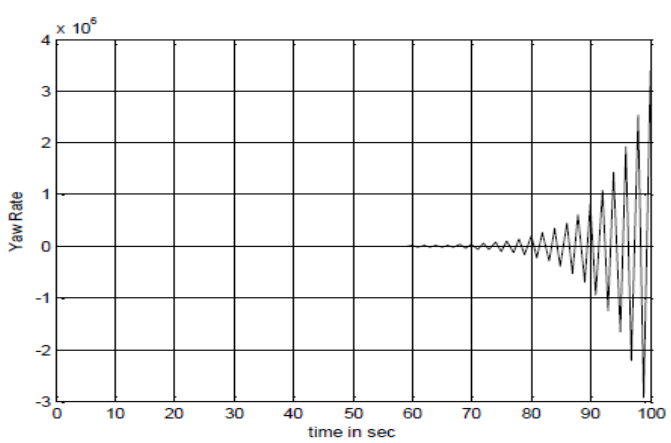

Figure 21 Variation of Yaw rate

Figure 2e shows that the high frequency rudder control does not seriously affect the heading if the sway velocity is used for rudder control (for the vessel moving at a speed of $13 \mathrm{~m} / \mathrm{s}$ or less). But figure $2 \mathrm{k}$ indicates the non-effectiveness of even the use of sway velocity on heading control above vessel speeds above $15 \mathrm{~m} / \mathrm{s}$. There is also significant increase in the roll angle at higher speeds.

\section{CONCLUSION}

The effect of cross coupling between sway, roll and yaw in the performance of RRCS has been discussed in this paper. The full state feedback for RRCS design is investigated and is seen that high frequency rudder motion used for roll reduction 
does not disturb the heading too much if the sway velocity is also taken for feedback.

Future developments can be done in several areas .In the simulation study performed, the ship model used is a linear one. A non-linear model may be used which can include the effect of additional linear and non-linear terms. In such a case , estimation can be done using Extended Kalman Filter. The use of non-linear controller such as the one presented by Chen,Shaw and Troesch[8] can also be used.

\section{REFERENCES}

[1] B.W King ,R.F Beek,and A.R Magee, Sea Keeping Calculation with Forward speed using time domain analysis, Proceedings of the $17^{\text {th }}$ symposium on Naval hydrodynamics,Hague, 1988,577-596.

[2] T.I.Fossen, Guidance and control of ocean vehicles, John Wiley and Sons , NewYork,1995.
[3] C.K.Chui and G.Chen, Kalman filtering with real time applications, New York: Springer-Verlag. 1991.

[4] Y.B.Shalom and X.R.Li, Estimation and tracking, Artech House Inc. 1993.

[5] R.E.Reid, A.Kemal and B.C. Mears, The use of wave filter design in Kalman filter state estimation of the automatic steering problem of a tanker in a seaway", IEEE trans. On Automatic Control ,Vol. AC - 29,July 1984,577-584.

[6] H.W.Sorenson, Kalman Filtering : Theory and application, IEEE press 1985.

[7] R.K.Bansal,A.K. Goel,and N.K Sharma, MATLAB and its applications in engineering,Pearson,India,2010.

[8] S.L Chen ,S.W Shaw,H.K Khalil, A.W Troesch, Robust stabilization of large amplitude ship rolling in regular beam seas, Non-linear dynamics and control,DE-Vol. 91,ASME, 1996,93-98. 